\title{
Reactions and diversity analysis of upland rice genotypes against blast disease of rice (Oryza sativa L.)
}

\begin{abstract}
Rice blast caused by the fungus Magnaporthe grisea is the most devastating rice disease and is severe in upland/dry land culture conditions. Fifty upland rice genotypes, including one resistant check, Pongsu Seribu-1 (PS-1), and one susceptible check, MR219, were evaluated to find out new sources of resistance and assess their diversity based on the reactions against M. grisea. The blast lesion type (BLT) and the percentage of diseased leaf area (\% DLA) were assessed following the standard evaluation system introduced by the International Rice Research Institute 7 days after inoculation. Diversity of the 50 rice genotypes was also analysed based on BLT and \% DLA. Resistant reactions were observed with the genotypes Biaw Bood Pae, Blau Noc, Chirikata 2, IPPA, IR 5533-50-1-10, IR 5533-55-1-11, Ja Hau, Ja No Naq, BR26, BRRI Dhan42, and BRRI Dhan43. The 50 genotypes were grouped into five clusters. Although the cluster analysis grouped the genotypes with greater similarity of their reactions, but it did not seem necessarily that all the clusters were formed according to the same reactions or origin. The higher range of inter-cluster distance indicated wider diversity among the populations. This information is useful for selecting suitable genotypes for hybridisation programmes for the development of blast-resistant varieties. The identified resistant genotypes could be used as new sources of resistance to develop durable blastresistant high-yielding upland rice varieties for challenging environments under water scarcity conditions, in addition to studying the mechanism of resistance in future breeding programmes.
\end{abstract}

Keyword: Blast disease; Diversity; Magnaporthe grisea; Susceptible; Upland rice 